\title{
Dimensiones de personalidad de marca. Caso de estudio: cadenas de farmacias
}

\author{
Colmenares, Oscar* \\ Saavedra Torres, José Luis**
}

\section{Resumen}

El presente artículo persigue tres objetivos: 1) determinar la personalidad de marca del sector Cadenas de Farmacias, 2) comparar los resultados obtenidos con estudios previos en Venezuela y 3) Validar los planteamientos de Austin, Siguaw y Mattila (2003). La investigación adoptó un muestreo no probabilístico accidental, para totalizar 295 muestras a través de la aplicación de un cuestionario vía entrevistas personales. Para el procesamiento de los datos se aplicó un análisis de Confiabilidad vía Alfa de Cronbach generándose las Dimensiones de Marca: Emocionante (0.91), Pasividad (0.81) y Sinceridad (0.83). La consistencia en los resultados obtenidos permite validar la posibilidad de obtener dimensiones de personalidad estables para una selección de productos y/o servicios a pequeña escala de forma estadísticamente robusta y a su vez permiten confirmar que si bien el modelo original de Aaker (1997) no es generalizable mundialmente, estudios nacionales individuales si representan un marco teórico y metodológico válido para determinar la personalidad en sectores e incluso marcas específicos.

Palabras clave: Marca, Personalidad de Marca, Rasgos de Personalidad, Cadenas de Farmacias.

\section{Dimension of the Brand Personality. Case Study: Pharmacy Chains}

\section{Abstract}

The present article pursued three objectives: 1) to determine the brand personality for the pharmacy chain sector, 2) to compare the results obtained through prior research in Venezuela and 3) to validate the statements of Austin, Siguaw y Mattila (2003). The research adopted a chance

Recibido: 11-09-06. Aceptado: 26-07-07

* $\quad$ Licenciado en Administración (Universidad Valle del Momboy, 2003). Estudiante de Maestría Gerencia Empresarial, Universidad del Zulia. Correspondencia Autor: oscolm29@yahoo.es

** Master en Administración (IESA, 1999), Profesor Asociado-Invitado de Mercadeo, FACESLUZ. Investigador acreditado al PPI: No. 7654. 
non-probability sampling consisting of 295 people by applying a questionnaire through personal interviews. Data was processed analyzing reliability with Cronbach's Alpha, generating the brand personality dimensions: Exciting (0.91), Passivity (0.81) and Sincerity (0.83). Consistency in the results allowed for validating the possibility of obtaining stable personality dimensions for a selection of products and/or small-scale services in a statistically robust manner and at the same time, confirmed that the original Aaker (1997) model cannot be generalized worldwide; however, individual national studies do represent a theoretical and methodological framework valid for determining personality in sectors and even for specific brands.

Key word: Brand, brand personality, personality traits, pharmacy chains.

\section{Introducción}

Las marcas se han constituido en la actualidad en una constante del mundo moderno. La marca desempeña un rol significativo en la identificación del producto y de acuerdo a sus características ayuda a los consumidores a identificarla plenamente y establecer relaciones cercanas entre ambos (Aaker, 1992; Keller, 1993; Belch y Belch, 1998; Stanton, et al., 2000; Tybout y Carpenter, 2002).

La investigación relacionada con la marca le ha ido infundiendo a su concepto diversas categorías de clasificación para poder entender su naturaleza. Entre estas categorías aparece desde principios de la década de los ochenta, las Personalidad de Marca (Haigood, 2001). Sung y Tinkham, (2005) definen este concepto como el significado simbólico que detenta un grupo específico de consumidores y que ve reflejado en las marcas sus valores y creencias compartidas.

La Marca y la Personalidad prestan una función simbólica o de auto-expresión para el individuo, por lo que le agregan significado a la vida del consumidor a través del status que le imprimen como socios de una relación cercana (Caprara, et al., 2001), convirtiéndose en la manera en la que perciben a los demás y a si mismos
(Keller, 1993). Al definir Personalidad de Marca como el "set de características humanas asociadas a la marca" (Aaker, 1997:347), se establece un vínculo entre los rasgos de personalidad de los individuos y los atribuidos a la marca; que se define al colocar las aptitudes, respuestas, emociones, entre otros; como eslabones intermedios que se sitúan entre las dimensiones y procesos entendidos como competencias de la personalidad (García, 2005; Arjona y Guerrero, 2004; Casanova, Herrera y Fernández, 2004).

En la evaluación de la conveniencia estratégica de crear o reforzar una clase particular de asociación de personalidad para una marca específica, se debe estudiar tanto la personalidad de marca, que en la actualidad los consumidores asocian con ella y con su competencia, así como la personalidad deseada y el grado de intensidad de dicho deseo por parte del segmento objetivo (Batra, et al., 2005). Estos análisis permiten evaluar la "diferenciación" que la asociación de marca tendrá y lo "relevante" de la misma. Tanto la "diferenciación" como la "importancia" son necesarias para que la asociación de personalidad de marca cree valor para el consumidor (Batra y Homer, 2004; Aufreiter, Elzinga y Gordon, 2003). 
Dimensiones de personalidad de marca

Colmenares, Oscar y Saavedra Torres, José Luis

Dada esta importancia estratégica, se hicieron diversos esfuerzos por encontrar un modelo de estudio de Personalidad de Marca, procurando desarrollar un instrumento confiable para ser utilizado a través de varias categorias de producto y segmentos, basándose en la extensa literatura sobre personalidad humana (Batra, et al., 2005). El modelo de estudio más representativo hasta la fecha de Personalidad de Marca (Azoulay y Kapferer, 2003:144), fue presentado por Aaker (1997). Este trabajo se ha adaptado a otras culturas y para realizar estudios correlaciones entre otras dimensiones de la marca, como los aspectos emocionales, imagen, entre otros (Rojas et al., 2004; Saavedra et al., 2005).

Sin embargo, existen opiniones encontradas, sobre si el modelo de Aaker (1997) es aplicable a aspectos específicos de marcas en particular o a sectores económicos concretos. Según Austin, Siguaw y Mattila (2003:89) "Es sumamente improbable que un esquema de investigación pueda ser desarrollado para que sea mundialmente generalizable a cualquier contexto en el cual la personalidad de marca o cualquier otro constructo relacionado con la marca deba ser medido".

De allí surge el objetivo del presente artículo, que es explorar el modelo de Personalidad de Marca en un sector económico específico, Cadenas de Farmacias, para: 1) Determinar la personalidad de marca de cada uno de las organizaciones que integran el sector, 2) Comparar los resultados provistos por Pirela et al., (2004) para encontrar similitudes entre los resultados de personalidad de marca a nivel nacional y los de sectores específicos y 3) Validar los planteamientos de Austin, Siguaw y Mattila (2003).
Para ello, se realizó un análisis teórico del modelo de Aaker (1997), haciendo énfasis en el proceso de recolección de datos propuesto, lo que permitió realizar una investigación fundamentada en principios empíricos-positivistas, con criterios de verdad basados en la obtención de datos generados por la aplicación de un cuestionario y en un tratamiento estadístico de la información a través del uso del análisis de correlación.

\section{Personalidad de Marca}

Desde 1958 han surgido diversas investigaciones orientadas a analizar a la Personalidad como un atributo de alta importancia para determinar el Valor de la Marca (Farquhar, 1989; Aaker, 1992 y 1996; Keller, 1993). Azoulay y Kapferer (2003:144) afirman "que las personas escogen las marcas de la misma forma como escogen a sus amigos, independientemente de sus habilidades y características físicas".

Aunque los conceptos de personalidad humana y de marca pueden ser similares, cada constructo es diferente, tanto en sus antecedentes, como en los papeles que desempeñan. Los Rasgos de personalidad humanos son creados y comunicados a través de otras vías, como lo son las características físicas, actitudes o comportamientos (Ambroise et al., 2004b). Es decir los rasgos de personalidad son deducidos directamente a través de otros elementos, no de ellos mismos. Por el contrario, la percepción de rasgos de personalidad de marca es deducida por el contacto directo o indirecto que los consumidores tienen con dichas marcas. La marca posee una rela- 
ción íntima con el consumidor que es asociada con rasgos de personalidad, no por vía natural, sino por los esfuerzos de comunicación realizados por los departamentos de mercadeo (Merunka et al., 2004).

Los gerentes de mercadeo confían en que la imagen del usuario de la marca o el set de características humanas que le son endosadas a personas famosas (p.ej. actores); puedan ser traspasado a los atributos de producto, a través de símbolos o de cualquier otro medio de personificación para de esa forma desarrollar asociaciones de personalidad de marca (Batra et al., 1993). Contrariamente al proceso de diferenciación, donde los atributos del producto son principalmente funcionales, el desarrollo de la personalidad de marca tiende a tener una función simbólica y de autoexpresión (Keller, 1993).

La personalidad de marca y la personalidad humana comparten de diversas semejanzas: ambas son duraderas y pueden, al menos en condiciones dadas, ayudar a explicar y predecir las acciones de los consumidores del segmento objetivo (Merunka et al., 2004).

\section{Modelos de Estudios de Personalidad de Marca}

El estudio de la medición de la personalidad de marca y el análisis de la construcción de un modelo para su medición ha sido largamente estudiado en los últimos 20 años (Batra et al., 2005).

Allen y Olson (1995) usaron un método que emplea el discurso narrativo para entender antecedentes y consecuencias de la personalidad de marca. Este estudio hizo énfasis en analizar la personalidad de marca desde el punto de vista corporativo, siendo el primer modelo que presenta una metodología cualitativa al estudio de marcas específicas (Kernstock y Srnka, 2002).

Por su parte, Caprara et al. (1997) estudiaron la personalidad a través del análisis de metáforas en un esquema lexicográfico que les permitió generar un modelo de cinco factores para identificar los principales atributos de la personalidad de marca.

Sin embargo, no es hasta Aaker (1997) cuando se desarrolla una escala generalizable para medir la personalidad de marca (Koebel y Ladwein, 1999). Aaker (1997) sistematizó la variable Personalidad de Marca a través de las cinco grandes dimensiones humanas (calidez, energía, aceptación, sociabilidad y actividad), desarrollando una estructura interna de cada una constituida por distintivos rasgos de personalidad.

Basada en el concepto de que "la personalidad de la marca incluye rasgos de personalidad humana como sentimentalismo, calidez y preocupación" (Aaker, 1997:347); la autora construyó grupos de los rasgos de personalidad, para constituir una dimensión. El estudio determinó la Personalidad de Marca de los consumidores estadounidenses, donde cada una de las dimensiones está conformada por un grupo de rasgos de personalidad distintivo. Las dimensiones encontradas fueron: Sinceridad, Emocionante, Competente, Sofisticación y Rudeza (Aaker, 1997).

El impacto del modelo de Aaker (1997) ha sido de tal magnitud en la comunidad académica del mercadeo, que según Azoulay y Kapferer (2003:144) "la gran mayoría de las investigaciones del 
Dimensiones de personalidad de marca

Colmenares, Oscar y Saavedra Torres, José Luis

área se han desarrollado usando este esquema". Hasta los momentos se han publicado siete estudios de ámbito nacional: Francia (Koebel y Ladwein, 1999), Venezuela (Barrios y Massa, Cristina 1999; Pirela et al., 2004); Japón y España (Aaker Benet-Martínez y Garolera, 2001), México (Álvarez-Ortiz y Harris, 2002), Rusia (Supphellen, y Gronhaug, 2003) y Korea (Sung y Tinkham, 2005) con resultados diversos.

A pesar del reconocimiento de la metodología de Aaker (1997), él mismo no ha estado exento de críticas. El principal cuestionamiento radica en las bases conceptuales, sobre si los aspectos que en el modelo están siendo medidos realmente representan "la personalidad" (Azoulay y Kapferer, 2003; Caprara et al., 2001). Empíricamente, autores se han quejado que el modelo de Aaker (1997) no se reproduce de igual manera en otros países y con otras muestras, especialmente cuando es usado para medir diferencias de personalidad de marca dentro de una categoría específica (Austin, et al., 2003). De igual forma, también se ha sido indicado que algunos reactivos de la escala de personalidad de marca, aparecen, según la categoría, para recoger características más bien funcionales de producto que de personalidad de marca.

En paralelo a estas críticas, la metodología de Aaker (1997) a sido empleada en diversos estudios sectoriales (Siguaw et al., 1999; Kim, 2000; Kernstock y Srnka, 2002, D’Astous y Levesque, 2003; Rojas et al., 2004; Saavedra et al., 2005), de ampliación de líneas (Diamantopoulos et al., 2005), análisis en la lealtad del cliente (Kim et al., 2001), entre otros.
Finalmente el último Modelo para la medición de la Personalidad de Marca, fue presentado por Merunka et al. (2004), donde se aplicó la escala de personalidad humana creada por Saucier a las marcas en un contexto multicultural, validando otros esquemas y escala de rasgos de personalidad.

\section{Fases del Estudio}

Se estructuraron cuatro etapas en el procedimiento de la investigación: 1) Desarrollar el estudio con los 22 rasgos de personalidad presentados por Pirela et al. (2004); 2) Selección del sector económico de referencia; 3) Diseño de un instrumento de recolección de datos y 4) Selección de la muestra. El orden de estas etapas fue vinculante y para pasar de una a otra era necesario haber cumplido los pasos y procedimientos de la etapa anterior.

\subsection{Selección de los rasgos de Personalidad de Marca}

El primer paso para la realización del presente estudio fue determinar los rasgos de Personalidad de Marca, para ello el modelo original de Aaker(1997), se desarrolló en el proceso siguiente:

1. Se hizo una clasificación en tres grupos: marcas cuya utilización tiene un contenido Simbólico (perfumes), contenido Utilitario (crema dental) y contenido Simbólico-Utilitario (automóviles). El proceso de agrupamiento (Clustering) determinó nueve grupos de cuatro marcas cada uno, con el criterio de debían representar cada una de las categorías (Aaker, 1997). 
2. Para determinar los rasgos de personalidad se usaron tres fuentes: escalas usadas en psicología y mercadeo, y una investigación cualitativa. Se generaron 114 rasgos de personalidad (Aaker, 1997).

3. La muestra de 1200 individuos del estudio de Aaker (1997) se determinó a través de cinco dimensiones demográficas del censo estadounidense. Los Instrumentos evaluaban los 114 rasgos en cuatro marcas (cluster), en una escala tipo Likert y se empleó el correo como método de recolección. Se obtuvo una tasa de retorno de 55\% (Aaker, 1997).

4. El análisis de los datos con Análisis Factorial, contó con una matriz de correlación de los rasgos (114) y las marcas (37), para luego promediarlos entre los sujetos (631).

5. Se utilizó el Análisis Factorial para determinar la confiabilidad de cada dimensión, resultando 15 facetas asociados a cada dimensión. El proceso de Clustering redujo los rasgos de 114 a 45 (Aaker, 1997).

6. Luego de correr las pruebas de confiabilidad de Test-Retest (correlación de Pearson y el Alpha de Cronbach) se obtuvieron indicadores que refleja- ban altos niveles de correlación interna. Tres rasgos con promedios menores a 0.60 fueron eliminados y la lista se redujo a 42 (Aaker, 1997).

Los estudios nacionales precedentes utilizaron de forma diferenciada el esquema de Aaker (1997). Para los estudios de Venezuela (1999); Japón (2001), España (2001) y Korea (2005) se duplicó la metodología de Aaker. Mientras que los estudios de Francia (1999), México (2002), Rusia (2003) y Venezuela (2004); se emplearon directamente los 42 rasgos de personalidad obtenidos por Aaker (1997) en la investigación original o se adaptó un modelo mucho más simple, al reducir el número de rasgos a una cantidad menor.

Para la presente investigación se seleccionaron 22 rasgos de personalidad luego de una depuración doble de dos criterios: presencia en al menos dos estudios previos y la mayor carga en la prueba de Análisis Factorial. En la selección final, se determinó que para la dimensión mexicana de "Género", sus rasgos (masculino y femenino) están incluidos dentro de otras dimensiones, por lo que no se incluyó directamente. Los rasgos seleccionados para el presente estudio se presentan en el Cuadro 1.

\section{Cuadro 1. Rasgos de Personalidad seleccionados}

\begin{tabular}{ccc}
\hline $\begin{array}{c}\text { Rasgos presentes en todos } \\
\text { los estudios nacionales }\end{array}$ & $\begin{array}{c}\text { Rasgos comunes en al menos } \\
\text { dos (2) estudios nacionales }\end{array}$ & $\begin{array}{c}\text { Propios del Estudio } \\
\text { de España }\end{array}$ \\
\hline atrevido, divertido, & sofisticado, amigable, & impulsivo, \\
chévere, moderno, & tranquilo, tímido, & apasionado, \\
pacifico, elegante, & emocional \\
orientado a la familia, & julento, & \\
sincero, confiable, & juvenil & \\
seguro de sí mismo, & & \\
femenino, masculino, & & \\
fuerte de carácter & & \\
\hline
\end{tabular}

Fuente: Elaboración propia (2006). 
Dimensiones de personalidad de marca

Colmenares, Oscar y Saavedra Torres, José Luis

\subsection{Selección del Sector Económico de Referencia}

Se seleccionó el sector farmacéutico como la referencia para el presente estudio, ya que los rankings de estudios sindicados sobre marcas con mayor valor de diversos sectores, suelen presentar de forma constante tres sectores de actividad económica: consumo masivo, entretenimiento y farmacéutico (Sweeney y Swait, 1999).

Dado que el sector farmacéutico incluye una alta diversidad de actores, se seleccionó específicamente a las Cadenas de Farmacias, ya ellas permiten validar la revisión teórica al catalogarlas como un producto altamente informativo pues forman parte de las marcas de contenido Simbólico-Utilitario, al combinar altos niveles de servicio y altos niveles de producto tangible, y que son catalogados como altamente representativos por el estudio original de Aaker (1997). Adicionalmente existe referencia de estudios previos que han utilizado canales de comercialización para estudiar las dimensiones de personalidad de marca con resultados positivos (Kernstock y Srnka, 2002 y D'Astous y Levesque, 2003).

Por razones de conveniencia y basados en información estadística de campo, se determinaron las cadenas de farmacia según cuatro criterios: presencia nacional, cadena regional con trayectoria reconocida, cantidad de establecimientos y presencia masiva y constante en los medios de comunicación. Al analizar información del sector suministrada por la Asociación de Cámaras de Farmacia de Venezuela, así como revistas especializadas en el área de negocios (Producto y
Dinero), quedaron seleccionadas las cadenas: Farmatodo, Farmapunto, Farmacias SAAS y Locatel.

\subsection{Diseño del instrumento}

El instrumento se diseñó inicialmente con 12 ítems, con preguntas de recolección de datos demográficos, preguntas de control sobre la imagen y utilización de cadenas de farmacias y las específicas de personalidad de marca.

Para las preguntas de personalidad de marca se elaboró una matriz donde se encontraban los 22 rasgos de personalidad de marca del estudio de Pirela et al. (2004) y cada una de las cadenas de farmacias seleccionadas. En esta matriz los encuestados debían responder cual o cuales rasgos de la personalidad describían mejor a cada cadena de farmacias, empleando para ello una escala tipo Likert, de cinco opciones de respuesta, donde uno era "Para nada" y cinco "Muchísimo".

\subsection{Diseño de la Muestra}

Debido a la inexistencia de bases de datos o record de consumidores de las cadenas de farmacias y a la necesidad de obtener información de "antes" que los consumidores tomen la decisión definitiva de ir a una farmacia, para evitar los sesgos naturales de responder un cuestionario en el sitio que se está evaluando (Zikmund, 1998; Kinnear, 1999 y Benassini, 2001); se utilizó la técnica de muestreo no probabilístico accidental (Sabino 2000). El instrumento se aplicó a los pacientes que acudieron a centros asistenciales de consulta privada y pública ubicados en la Parroquia Olegario Villalo- 
bos $^{1}$ del Municipio Maracaibo del Estado Zulia en horario comprendido de $8 \mathrm{AM}$ a $12 \mathrm{M}$, y de 2 a $6 \mathrm{PM}$, los días lunes, martes y miércoles, ya que según los departamentos de administración de los centros seleccionados eran los días de mayor afluencia de pacientes.

La muestra quedó constituida por 295 personas entrevistadas. El instrumento fue aplicado en el mes de Diciembre de 2005, a través de entrevistas personales en las salas de espera de consulta externa.

\section{Resultados}

Para las dimensiones de personalidad se sumaron los puntajes en cada rasgo de personalidad y por cada cadena de farmacia de estudio, obteniendo así las medias correspondientes. Se corrió el Análisis Factorial para cinco, seis, y siete factores (carga factorial de 0.5); obteniéndose como óptimo agrupaciones de rasgos de personalidad en tres factores. Las dimensiones encontradas fueron: Emocionante, Sinceridad y Pasividad.

En el análisis de confiabilidad de cada factor se utilizó el indicador Alfa de Cronbach y se encontró que los rasgos que componen las dimensiones: Emocionante (0.91), Pasividad (0.81) y Sinceridad (0.83) son explicativos de estas dimensiones. Se corrió la prueba de confiabilidad del constructo de personalidad de marca, usando las tres dimensiones y se encontró que éstos son explicativos del constructo al obtener un alfa de 0.82 .

\section{Conclusiones}

La presente investigación se planteó tres objetivos al momento de explorar el modelo de Personalidad de Marca en el sector Cadenas de Farmacias: 1) Determinar la personalidad de marca de cada uno de las organizaciones que integran el sector, 2) Comparar los resultados provistos por Pirela et al. (2004) para encontrar similitudes entre los resultados de personalidad de marca a nivel nacional y los de sectores específicos y 3) Validar los planteamientos de Austin, Siguaw y Mattila (2003). Para hacer una revisión detallada de cada uno, se presentan a continuación conclusiones específicas por cada objetivo.

\subsection{Dimensiones de Personalidad}

Los rasgos y las dimensiones obtenidas en el presente estudio permiten determinar la personalidad de marca del sector de las cadenas de farmacia. Los rasgos de personalidad incluidos en cada dimensión se presentan en el Cuadro 2.

De igual forma, los resultados obtenidos permiten analizar cual de las dimensiones es más asociada con cada una de las marcas de cadenas de farmacias analizadas. El Cuadro presenta las dimensiones asociadas con cada cadena de farmacia y los rasgos más significativos para cada una

El hecho que en los resultados particulares de las cadenas de farmacias analizadas no aparezcan todas las

1 Esta parroquia aglutina los centros asistenciales de mayor demanda: Hospital Coromoto, Centro Médico de Occidente, Policlínica Maracaibo, Policlínica Amado, Hospital Central. 
Dimensiones de personalidad de marca

Colmenares, Oscar y Saavedra Torres, José Luis

dimensiones y rasgos obtenidos para el sector, permite validar los supuestos teóricos de Aaker (1997) donde expone que el set de dimensiones son el abanico de opciones de personalidad para interpretar una marca, más sin embargo, al igual que en el aspecto humano, los seres humanos no asocian toda la gama de dimensiones de personalidad con una sola persona, sino que asignan características particulares, y por ende rasgos específicos para cada una. En el caso de las marcas el mecanismo actúa de la misma manera, consiguiéndose entonces que los consumidores relacionan un grupo pequeño de rasgos con marcas específicas.

\subsection{Comparación con estudios anteriores}

Al analizar los resultados obtenidos por Pirela et al. (2004), se consiguió consistencia entre las dimensiones reflejadas por ambos estudios, lo que demues- tra y valida la hipótesis del trabajo central de Aaker (1997) que postula que todas las marcas comercializadas en el país estudiado van a ser percibidas, analizadas y seleccionadas por los consumidores a través del set de dimensiones y rasgos determinado a nivel nacional.

Los resultados mostrados en la Cuadro 3, permiten comparar los resultados de el estudio de las dimensiones de personalidad de marca para Venezuela llevado a cabo porPirela et al. (2004) y los obtenidos en el presente estudio.

Esta consistencia en los resultados obtenidos utilizando objetos de estudio diferentes (centros comerciales y cadenas de farmacias) y procedimientos de recolección de datos distintos (encuestas por internet y auto-administradas en un estudio, y cuestionarios inducidos en el otro) permite, por una lado, validar la posibilidad de obtener dimensiones de personalidad estables para una selección de productos y/o servicios pequeña o individual (p. ej. Cadenas de farmacias), que

\section{Cuadro 2. Dimensiones de Personalidad de Marca en Cadenas de Farmacias}

\begin{tabular}{cccc}
\hline Dimensiones & Sinceridad & Emocionante & Pasividad \\
\hline Rasgos de & Honesto & Actual & Práctico \\
Personalidad & & $\begin{array}{c}\text { Entusiasta } \\
\text { Encantador }\end{array}$ & \\
& & & \\
\hline
\end{tabular}

Fuente: Propia (2006).

\section{Cuadro 3. Dimensiones y rasgos de Personalidad de Marca en Cadenas de Farmacias}

\begin{tabular}{ccccc}
\hline & Locatel & Farmacias SAAS & Farmatodo & Farmapunto \\
\hline Dimensiones & Emocionante & Sinceridad & $\begin{array}{c}\text { Sinceridad y } \\
\text { Pasividad }\end{array}$ & Pasividad \\
$\begin{array}{c}\text { Rasgos más } \\
\text { resaltantes }\end{array}$ & Actual & Confiable & $\begin{array}{c}\text { Confiable y } \\
\text { Práctico }\end{array}$ & Práctico \\
\hline
\end{tabular}

Fuente: Propia (2006). 


\section{Cuadro 4. Comparación de las Dimensiones de Personalidad encontradas}

\begin{tabular}{ccc}
\hline & \multicolumn{2}{c}{ Alfa de Cronbach del estudio } \\
\cline { 2 - 3 } Dimensiones de Personalidad & Pirela et al. (2004) & Colmenares y Saavedra (2006) \\
\hline Emocionante & 0.91 & 0.91 \\
Sinceridad & 0.83 & 0.81 \\
Pasional & 0.88 & - - \\
Pasividad & 0.76 & 0.88 \\
Rudeza & 0.68 & $-\ldots$ \\
\hline
\end{tabular}

Fuente: Propia (2006).

sean internamente robustas estadísticamente y que a su vez formen parte del set genérico de dimensiones y rasgos que definen la identidad nacional del consumidor.

Adicionalmente, los resultados obtenidos permiten confirmar la validez de una investigación basada en la depuración de los rasgos de personalidad de Aaker (1997), al pasar de los 42 originales a los 22 preseleccionados para el caso venezolano por Pirela et al. (2004), generando información estadísticamente robusta y metodológicamente válida.

\subsection{Validar la el planteamiento de Austin, Siguaw y Mattila (2003)}

Según Austin, Siguaw y Mattila (2003:89) "es sumamente improbable que un esquema de investigación pueda ser desarrollado para que sea mundialmente generalizable a cualquier contexto en el cual la personalidad de marca o cualquier otro constructo relacionado con la marca deba ser medido".

Los datos aportados en la presente investigación permiten concluir que validamos la hipótesis de Austin, Siguaw y
Mattila (2003) que afirma que los resultados del modelo de Aaker (1997) no son generalizables a nivel mundial, ya que los resultados obtenidos en estudios nacionales por separado, así como sus subsiguientes comparaciones han demostrado la existencia de diferencias sustanciales entre ellos, denotando que el factor cultural y el medio ambiente particular de cada país genera condiciones propias y únicas de asociación de marca.

Esta conclusión se ve respaldada por la publicación de Gita Venkataramani, et. al., (2005) quienes analizan y comparan otra vía para determinar la Personalidad de Marca además de los rasgos de personalidad: Las impresiones medidas a través de las inferencias evaluativas. Esta investigación tuvo como objetivo hacer el primer esbozo de reflejar la situación bidireccional y dinámica que existe entre las actuales asociaciones de marca y la información de mercadeo generada para apoyar e impulsar la marca.

Por otra parte, el presente estudio permite concluir, que si bien el estudio original no es generalizable a nivel mundial, los estudios nacionales si pueden servir como marco teórico-metodológico para, 
Dimensiones de personalidad de marca

Colmenares, Oscar y Saavedra Torres, José Luis

no sólo medir personalidad de marca de sectores específicos así como de otros constructos relacionados con la marca. Esta conclusión rechaza el segundo planteamiento de Austin, Siguaw y Mattila (2003:89) que afirman que "a pesar del hecho que Aaker (1997) propone usos para su modelo en los que se pudiese medir y analizar la personalidad de marca a nivel individual, el esquema de medición de la personalidad no es generalizable a marcas individuales dentro de una categoría de producto".

\section{Referencias Bibliográficas}

Aaker, David (1992). "The value of brand equity". Journal of Business Strategy. Vol. 13, No. 4, USA. Pp. 27-32.

Aaker, David (1996). Building Strong Brands. New York, USA. The Free Press. Pp. 400.

Aaker, Jennifer (1997). "Dimensions of Brand Personality" Journal of Marketing Research. Vol. 34. Chicago, USA. Pp. 347-356.

Aaker, Jennifer; Benet-Martínez, Verónica y Garolera, Jordi (2001). "Consumption Symbols as Carriers of Culture: A Study of Japanese and Spanish Brand Personality Constructs". Journal of Personality \& Social Psychology. Vol. 81. No. 3. USA. Pp. 492508.

Allen, D. y Olsen, J. (1995) "Conceptualizing and Creating Brand Personality: A Narrative Theory Approach". Advances in Consumer Research. No. 22.

Álvarez-Ortiz, Cecilia y Harris, J. (2002). "Assessing the Structure of Brand Personality among Global and Local Mexican Brands". 2002 AMA Summer Educators Conference. San Diego, CA, USA, 2002.
Ambroise, L.; Ferrandi, J.M.; Merunka, D. y Valette-Florence, P. (2004a). "Development of a Brand Personality Scale and Application to Two French Supermarket Brands". 2004 Society For Marketing Advances. Annual Conference and VII Retail Strategy and Consumer Decision Research Symposium. San Petersburgo, Florida (USA) 2-6 Noviembre.

Ambroise, L.; Ferrandi, J.M.; Merunka, D. y Valette-Florence, P. (2004b). "How Well Does Brand Personality Predict Brand Choice? A Measurement Scale and Analysis Using Binary Regression Models". Asian Pacific Advance in Consumer Research. Vol. 6. Pp. 30-38.

Arjona, José Francisco y Guerrero, Susana (2004). "Un Estudio sobre los estilos y estrategias de Afrontamiento y su relación con la variable Adaptación en Adolescentes de Secundaria". Málaga, España. Http://www. psicologiacientifica.com/publicaciones/biblioteca/articulos/ar-arjona01.htm. Revisado, Agosto 2005.

Aufreiter, Nora A., Elzinga, David y Gordon, Jonathan W. (2003). "Better Branding". McKinsey Quarterly. Vol. 4. Pp. 2939.

Austin, Jon R; Siguaw, Judy A. y Mattila, Anna S. (2003). "A Re-examination of the Generalizability of the Aaker Brand Personality Measurement Framework". Journal of Strategic Marketing. Vol. 11. Junio. Pp. 77-92.

Azoulay, Audrey y Kapferer, Jean-Noel (2003). "Do brand personality scales really measure brand personality?" Journal of Brand Management. Vol. 11, No. 2. USA. Pp. 143-155.

Barrios, Marianela y Massa, Cristina (1999). "Dimensiones de la personalidad de la marca en Venezuela" (Tesis de Maestría, Instituto de Estudios Superiores de Administración). 
Batra, R.; Lehmann, D. y Singh, D. (1993) “The Brand Personality Component of Brand Goodwill: Some Antecedents and Consequences" en Brand Equity and Advertising. Ed. Por Aaker y Biel. Hillsdale, NJ, USA. Lawrence Erlbaum Associates.

Batra, Rajeev y Homer, Pamela M. (2004). "The Situational Impact of Brand Image Beliefs". Journal of Consumer Psychology. Vol. 14. No. 3. Pp. 318-330.

Batra, Rajeev; Lenk, Peter y Wedel, Michael (2005). "Separating Brand from Category Personality". University of Michigan. Working Paper. http://webuser.bus.umich.edu/plenk/Brand\%20P ersonality.pdf Revisado, Agosto 2006.

Belch, George y Belch, Michael (1998). Advertising and Promotion. 4ta. Edición. USA. Irwin/McGraw-Hill. Pp. 762.

Benassini Marcela (2001). Introducción a la Investigación de Mercados. Prentice Hall. México.

Caprara G.; Barbaranelli C.; y Guido G. (1997) "Personality as Metaphor: Extension of the Psycho-lexical Hypothesis and the Five Factor Model to Brand and Product Personality Description". European Advances in Consumer Research. Vol. 3. Pp. 61-69.

Caprara, G. V., Barbaranelli, C., y Guido, G. (2001). Brand personality: "¿How to make the metaphor fit?" Journal of Economic Psychology. Vol. 22. Pp. 377-395.

Casanova, Caridad Luisa; Herrera, Luis Felipe y Fernández Berrocal, Pablo (2004). La Estructura de la Inteligencia Emocional en pacientes portadores de trastornos Neuróticos. http:// www.monografias.com/trabajos20/ pacientes-neuroticos/pacientes-neuroticos.shtml. Revisado, Agosto 2005.

D’Astous, Alain y Levesque, Mélaine (2003). "A scale for measuring store persona- lity". Psychology \& Marketing. Vol. 20, No. 5. USA. Pp. 455-469.

Diamantopoulos, Adamantios; Smith, Gareth y Grime, lan (2005). "The impact of brand extensions on brand personality: experimental evidence". European Journal of Marketing. Volume 39. Numbers 1-2. Pp. 129-149.

Farquhar, Meter (1989). "Managing brand equity". Marketing Research. Vol. 1, No. 3. USA. Pp. 24-33.

García, Claudia (2005). Rasgos de Personalidad en Deportistas Campeones Nacionales Mexicanos. México D.F http://64.233.179.104/search?q=cache:njqSwv01tawJ:www.lustrados. Com/documentos/presonalidaddep mex.doc+personalidad+emocional+ correlacion\&hl=es Revisado, Agosto 2005.

Haigood, Traci L. (2001). "Deconstructing Brand Personality". 2001 AMA Summer Educators Conference. Pp. 327-328.

Gita Venkataramani, Johar; Sengupta, Jaideep y Aaker, Jennifer (2005): "Two roads to updating brand personality impressions: Trait versus evaluative inferencing", Journal of Marketing Research. Vol. 42. No. 4. Pp. 458 - 469.

Keller, Kevin (1993). "Conceptualizing, measuring, and managing customer-based brand equity". Journal of Marketing. Vol. 57 No. 1. USA. Pp. 1-22.

Kernstock, Joachim y Srnka, Katharina. (2002) "Brand personality-management: an integrative approach to corporate brand-management considering internal and external stakeholders" AFMIRM Conference "Brands, Branding and Brand Equity" Paris, Francia.

Kim (2000): "Examination of brand personality and brand attitude within the apparel product category", Journal of Fashion Marketing and Management. Vol. 4. No. 3. Pp. 243-252. 
Dimensiones de personalidad de marca

Colmenares, Oscar y Saavedra Torres, José Luis

Kim, Han y Park (2001): "The effect of brand personality and brand identification on brand loyalty: Applying the theroy of social identification". Japanese Psychological Research. Vol. 43 no. 4, pp. 195-206.

Kinnear, Thomas (1999). Investigación de Mercados Un enfoque Aplicado. 5ta. Edición. México. Editorial Mc Graw Hill. Pp. 874.

Koebel, Marie-Noëlle y Ladwein, Richard (1999). "L'échelle de personalité de la marque de Jennifer Aaker: Adaptation au contexte français". Decisions Marketing. No. 18. Paris, Francia. Pp. 81-88.

Merunka, D.; Ferrandi, J. M.; De Barnier, V. y Valette-Florence P. (2004) "How Well does a Human Personality Scale apply to a Brand?" Asian Pacific Advance in Consumer Research. Vol. 5. Pp. 53-60.

Pirela, José Luis; Villavicencio, Héctor y Saavedra T., José Luis (2004). "Dimensiones de Personalidad de Marca. Estudio Exploratorio de los Rasgos de Personalidad en Venezuela". Revista de Ciencias Sociales. Vol. X, No. 3. Sep. - Dic. Maracaibo, Venezuela. Pp. 430 - 440.

Revista Dinero (2006). "Estudio del sector farmacéutico". http://www.dinero. com.ve/monte/farmacia.html Revisado Febrero, 2006.

Revista Producto (2006). "El negocio de turno: Farmacias". http://www.producto.com.ve/238/notas/portada.html Revisado Febrero, 2006

Rojas-Méndez, Podlech y Silva-Olave (2004). "The Ford brand personality in Chile". Corporate Reputation Review. Vol. 7. No. 3. Pp. 232-251.

Saavedra Torres, José Luis (2004). "Capital de Marca desde la Perspectiva del Consumidor". Revista Venezolana de Gerencia. Año 9. No. 27. Maracaibo, Venezuela. Pp. 508-530.
Saavedra, José Luis; Pirela, José Luis y Villavicencio, Héctor (2005). "Correlación entre Dimensiones de Personalidad de Marca y la Marca Emocional. Estudio en Centros Comerciales". Revista Ciencia Administrativa. Universidad Veracruzana. México. No. 1. Págs. 128-139.

Sabino, Carlos (2000). Metodología de la Investigación. Caracas. Epistemi.

Siguaw, Mattila y Austin (1999): "The brand personality scale. An application for restaurants". Cornell Hotel and Restaurant Administration Quaterly. June. Pp. 48-55.

Stanton, William; Etzel, Michael y Walker, Bruce (2000). Fundamentos de Marketing. 11ra Edición. México. McGrawHill Interamericana.

Sung, Yongjun y Tinkham, Spencer F. (2005). "Brand Personality Structures in the United States and Korea: Common and Culture-Specific Factors". Journal of Consumer Psychology. Vol. 5. No. 4. Pp. 334-350.

Supphellen, Magne y Gronhaug, Kjell (2003). "Building foreign brand personalities in Russia: The moderating effect of consumer ethnocentrism". International Journal of Advertising. Vol. 22. No. 2. Eastbourne, Inglaterra. Pp. 203.

Sweeney, Jilian y Swait, Joffre (1999). "Brand Equity: An Integrated Framework". Australian and New Zealand Marketing Academy Conference (ANZMAC). School of Marketing, University of New South Wales. Pp. 1-7.

Tybout, Alice y Carpenter, Gregory (2002). "Crear y Administrar Marcas" en Marketing según Kelloggs. Ed. por lacobucci, Dawn. España. Vergara Editores/Business.

Zikmund, William (1998). Investigación de Mercados. 6ta. Edición, México. Editorial Prentice Hall. 